\title{
A Near Infrared Spectroscopy-Based Test of Calf Muscle Function in Patients with Peripheral Arterial Disease
}

\author{
Brian Lindegaard Pedersen, MD ${ }^{1} \quad$ Niels Bækgaard, MD ${ }^{1} \quad$ Bjørn Quistorff, MD, DMSc ${ }^{2}$ \\ ${ }^{1}$ Department of Vascular Surgery, Rigshospitalet and Gentofte \\ Hospital, Copenhagen, Denmark \\ 2 Department of Biomedical Sciences, University of Copenhagen, The \\ Panum Institute, Copenhagen, Denmark \\ Address for correspondence Brian Lindegaard Pedersen, MD, \\ Department of Vascular Surgery, Rigshospitalet, Blegdamsvej 9, 2100 \\ Copenhagen $\emptyset$, Denmark (e-mail: Brian.Lindegaard. \\ Pedersen.01@regionh.dk).
}

Int J Angiol 2015;24:25-34.

\begin{abstract}
Background The study aims to test a new, simple, and reliable apparatus and procedure for the diagnostics and treatment evaluation of peripheral arterial disease (PAD). The test apparatus involves near infrared spectroscopy (NIRS) of a main part of the lower leg muscles during isometric flexion and extension of the ankle joint performed with the foot strapped in a specially designed pedal ergometer.

Design To evaluate the reproducibility of the new test compared with an existing testing method of treadmill walking.

Methods Eleven patients participated in the study: nine patients with claudication and two age-matched patients without claudication. Each patient was tested with an isometric ergometer pedal test and a treadmill test applying NIRS measurements of the anterior tibial and the gastrocnemius muscles (GAS). Tests were repeated three times with randomly selected intervals between individual test runs. Intraclass correla-

Keywords

- near infrared spectroscopy

- peripheral arterial disease

- reproducibility of test measurements

- pedal ergometry

- treadmill

- dynamic and isometric exercise test tion constant (ICC) was used to describe reproducibility. The ICC was calculated using the area under the NIRS oxygenated hemoglobin $\left(\mathrm{Hb}_{\mathrm{ox}}\right)$ curve, the initial velocity of the $\mathrm{Hb}_{\mathrm{ox}}$ recovery curve, force measurements, and walking time.

Results The ICC of the GAS was between 0.92-0.95 (foot-pedal) and 0.70-0.98 (tread mill) and of the anterior tibial muscle was between 0.87-0.96 (foot-pedal) and 0.670.79 (tread mill).

Conclusion In this study, we contribute a new apparatus and test protocol for peripheral arterial disease (PAD) applying NIRS technique and controlled physical activity to evaluate the degree of muscle oxygenation under specific functionally relevant conditions. Thus, we have developed a clinically applicable "easy-to-do" exercise test of patients with chronic PAD which show high reproducibility.
\end{abstract}

The objective of this study was to evaluate whether a new simple isometric near infrared spectroscopy (NIRS) foot pedal test of lower leg muscles could be used for functional testing of patients suffering from peripheral arterial disease (PAD) in a daily clinical setting as an alternative to the treadmill test now being used. In a comparison of the new test and the existing treadmill test, we applied NIRS recording in both tests. We hypothesized that a pedal test would have an equal or better reproducibility than a standard treadmill test because the pedal test was designed to reduce factors that influence the reproducibility.

The diagnosis and clinical staging of PAD is currently based on patient symptoms of muscle ischemia combined with ankle blood pressure (ankle brachial index [ABI]), published online

February 9, 2015
Copyright $\odot 2015$ by Thieme Medical Publishers, Inc., 333 Seventh Avenue, New York, NY 10001, USA. Tel: +1(212) 584-4662.
DOI http://dx.doi.org/ 10.1055/s-0035-1544223. ISSN 1061-1711. 
angiography, magnetic resonance angiography, computer tomography angiography, ultrasound duplex scanning, and other clinical findings. ${ }^{1}$ Unfortunately, the test results with these procedures are not always specific with respect to patient symptoms and clinical findings. This is most likely the result of different pain thresholds and perhaps also different daily-life activity levels among patients. The reported symptoms, such as reduced walking distance in claudication, are known to involve major intraindividual as well as interindividual variability and are not always a true reflection of the actual walking distance. ${ }^{1,2}$ In particular, in patient cases with claudication symptoms and a normal ABI, the need of a more appropriate functional exercise test has become apparent.

\section{Existing Functional Test of Peripheral Arterial Disease}

The existing functional tests of PAD involve either whole body level, such as a treadmill walking test, or isolated muscle group exercise of the lower extremities, such as the new pedal test described in this article. Both tests are conducted with measurements of pre- and postexercise ABI.

\section{The Treadmill Test}

Using whole body exercise-based tests introduces several problems. One problem is reduced walking time/distance by dysfunctions other than leg claudication, for example, cardiac angina, chronic obstructive lung disease, and mechanical disabilities of the legs, which was pointed out by Yamamoto et al. $^{3}$ In situations where other morbidities coexist with PAD, walking time will not be diagnostic specific of PAD because walking time/distance is also reduced by other symptoms than claudication. Nevertheless the treadmill could provide valuable information on what actually stops the patient from walking, the comorbidity or PAD. But this is difficult in situations where both diseases contribute equally to the walking disability. Treadmill testing is often impossible to perform in the practical clinical setting in patients with critical ischemia since most of them are typically not able to walk. Nevertheless, the treadmill test is probably the test procedure which most closely mimics the situation of real walking.

\section{The Pedal Tests}

With single muscle group exercise, such as a pedal test, some of the problems with the interference by other diseases could be avoided and the test, therefore, most directly evaluates the relation between muscle activity and blood supply. In the pedal test, this is achieved with the patient in a seated position and by reducing the strength of the muscle work which will reduce the influence of common comorbidities, such as, chronic obstructive pulmonary disease, cardiac angina, and mechanical disabilities.

This study presents a new apparatus and test. Various dynamic pedal protocols have been presented before, that is, Yamamoto et $\mathrm{al}^{3}$ and Cameron et $\mathrm{al}^{4}{ }^{4}$ both of whom used a test based upon pain evaluation during exercise. Yamamoto found reliability of 0.92 (Cronbach $\alpha$ value) for the same treadmill protocol as used in this study and 0.90 for a foot pedal test. Both values were obtained for maximal walking distance/exercise time. Lower reproducibility was found using pain-free walking and pain-free exercise time $(0.88$ and 0.66). NIRS was used in both tests although not reported in detail. Manning et al did not report reliability values but concluded that a dynamic pedal test with fixed duration ( 2 minutes/exercise frequency 60 per minute) had a higher sensitivity for PAD compared with a treadmill test. NIRS was not used in these studies and actually $33 \%$ of the patients did not produce a decrease in the post $\mathrm{ABI}$ measurements with the pedal test.

The above-mentioned studies reported less reproducibility in the situation with pain-free exercise and, therefore, we chose an isometric exercise protocol with fixed exercise duration of only 1 minute with maximal voluntary contraction (MVC) to insure a significant decrease in the NIRS parameters. This way we attempted to make the new test more clinically useful and less time consuming.

\section{Near Infrared Spectroscopy Measurements}

Measuring the ABI pre- and postexercise is time consuming and often there is a time delay between the end of the exercise and the postexercise $\mathrm{ABI}$ values, depending on the experience of the investigator, which certainly contributes to the reliability of the test.

NIRS measures oxygenation continuously in the relevant muscle noninvasively, which is a much simpler technology to implement compared with the ABI-based protocols. The actual technique works by emitting light by a laser optode in the near infrared spectrum $(800-900 \mathrm{~nm})$ through the skin and into the underlying muscle. The light is penetrating the tissue in all directions and is absorbed specifically by hemoglobin in the blood and myoglobin in the muscle. The receiving optode collects a minor fraction of the emitted light and the amount of light received depends primarily on the distance between the two optodes and the concentration of the specifically absorbing molecules in the blood and tissue. The difference in the absorbance of light of different wavelength is exploited to determine the relative oxygenation level of the measured tissue, since oxygenized and deoxygenized hemoglobin have different absorption spectra. The major part of the light received by the receiving optode will have traveled the shortest distance from the emitter to the receiver optode and in practical terms the penetration depth from which the signal is received is, roughly speaking, half the length of the distance between the light emitter and sensor ${ }^{5}$ (approximately $2 \mathrm{~cm}$ in this study). Measuring muscle oxygenation provides the balance between actual perfusion and muscle oxygen consumption. It is commonly known that muscle oxygen consumption depends on the muscle fitness of the patient. ${ }^{6}$ Therefore, NIRS measurement, in contradiction to ABI measurement, takes muscle fitness into account. Muscle fitness is one of the major factors contributing to the wide range of symptoms seen at any given resting ABI. Muscle mitochondrial function is also an important factor, especially in PAD where a reduction in mitochondrial function is seen. ${ }^{7}$

Treadmill NIRS measurements obtained in the gastrocnemius muscle (GAS) have previously been used on patients 
with PAD. These studies have shown high reproducibility and a high correlation with the degree of PAD. ${ }^{8,9}$ NIRS during treadmill testing has been shown to correlate with measurement of ABI. ${ }^{9}$

Testing of muscle force and oxygenation with a pedal and NIRS has been done earlier in several studies $3,10,11$ and a reduced cardiac stress compared with the treadmill test in patients with PAD has been shown., ${ }^{3,12}$

With this new test, we hope to be able to include muscular fitness into the diagnostics of PAD in a simple clinical applicable way instead of just looking at the blood pressures. It was our aim to develop a test that can give a more precise diagnosis and the degree of disease.

\section{Methods}

Patients: Eleven patients (seven men and four women) were recruited from the vascular outpatient clinic at Gentofte Hospital, Denmark, nine with claudication and two age-matched patients without claudication, for details see - Table 1. The distribution of the different patient types was chosen to mimic the normal distribution of referred patients in our outpatient clinic. PAD was defined according to the TASC II consensus report. ${ }^{1}$ All patients were also enrolled in a larger patient series with the purpose of investigating muscle energy metabolism in patients with combined PAD and diabetes type 2. Only reproducibility of measurements and feasibility of the pedal test are considered in this article.

In addition, a series of tests was done using one of the authors (BLP) as a subject to get a picture of reproducibility under the most optimal conditions.

Oral and written informed consent was obtained from all patients and the project was approved by the local ethical committee (approval nr: H-D-2007-0074).

All patients with claudication were nonsurgically treated at the time of investigation, following a program with exercise counseling and medical treatment with antiplatelet and cholesterol-lowering drugs ( $\boldsymbol{-}$ Table $\mathbf{1}$ ). The pedal-based exercise test was developed and compared with an existing test based upon the recording of walking distance on a treadmill. The pedal test and the treadmill test were performed on the same day with appropriate recovery time in between. Both tests were repeated three times on three different randomly selected days with a mean time of 22.6 days (mean, range 2-130) in between. No changes in exercise pattern of the patients were detected in the investigation period. All tests were done by the primary investigator.

The ergometer pedal instrument: The developed pedal instrument is based upon a pedal ergometer designed for a whole-body magnetic resonance scanner. ${ }^{13}$

The pedal is made of black polyoxymethylene (POM) plastic with a sole of soft material which follows the shape of the foot and is adjustable to both the feet and leg length. The pedal is attached to a base of hard plastic which again is fixed to a hospital examination bed with wheels and stabilizing legs to avoid unnecessary movement. The foot is attached with the Velcro straps. Between the rear side of the pedal and the pedal base, a brass stick with an incorporated strain gauge

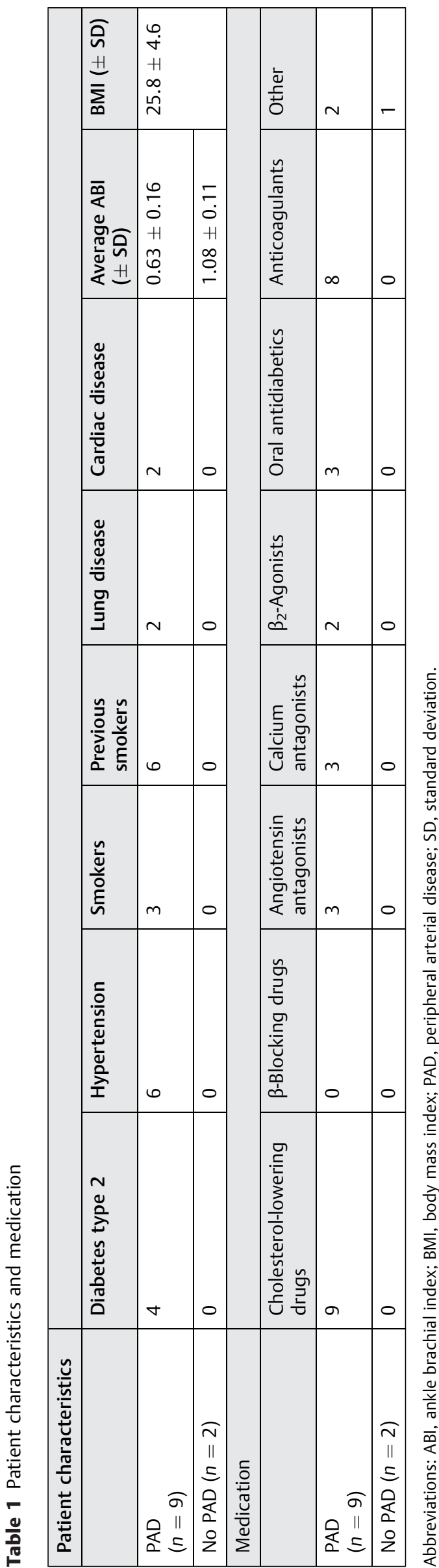



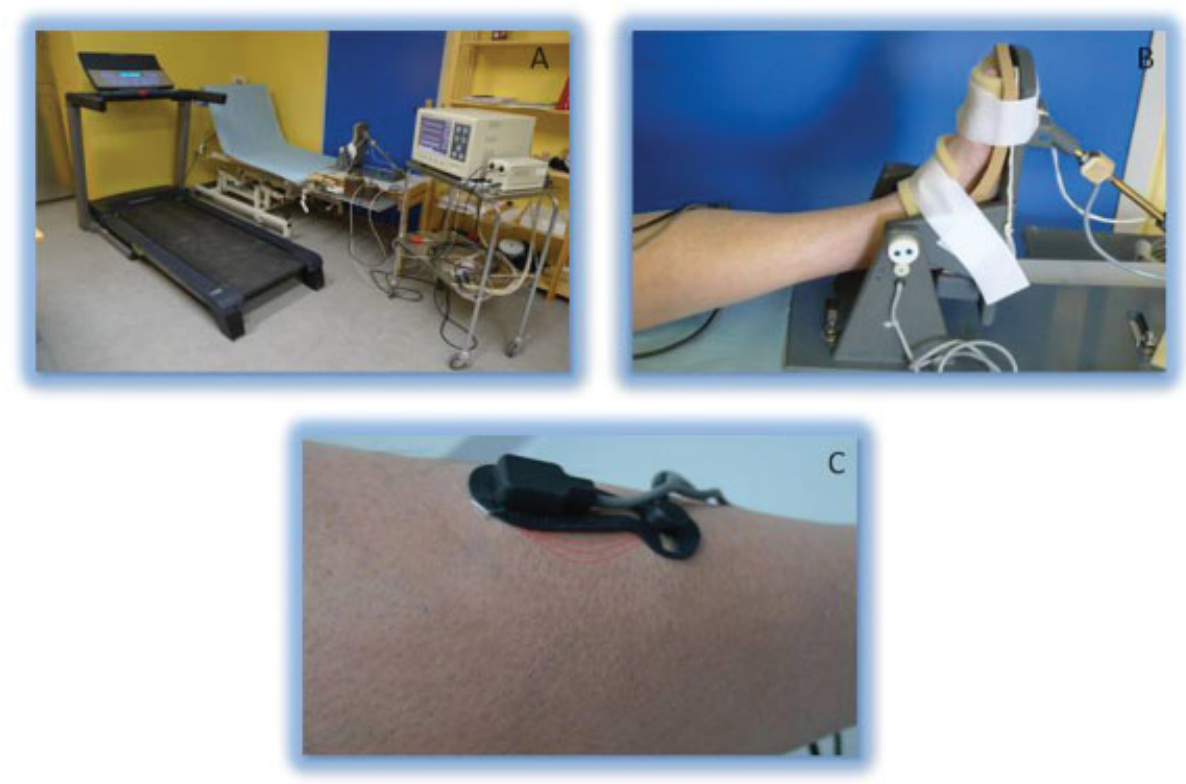

Fig. 1 The experimental setup (A) and the pedal instrument (B). The optode placement on the skin and sketch drawing of the light array (C).

in the middle is attached to obtain muscle force. Force is applied in both directions, allowing both the plantar flexion and extension to be studied. The pedal is fixed in place (90-degree position), allowing no movement. Data are obtained on a laptop using a sampling module (USB-1608FS) with TracerDAQ software (Measurement Computing, Norton, MA).

The patients were placed in a seated position with foot fixed to the pedal. The legs were placed with slight flexion in the knee joint (10 degrees) to allow optimal contraction of the calf muscles (individual adjusted).

The patients were resting for 3 minutes. This was the time needed to stabilize the NIRS signal. Next, a 1 minute plantar flexion (MVC) was made. NIRS measurements were continued for a period of 7 to 10 minutes until recovery. The exercise protocol was repeated with dorsal flexion after appropriate recovery.

NIRS measurements: A NIRO-200 spectrometer (Hamamatsu, Japan) was used to obtain oxygenated $\left(\mathrm{Hb}_{\mathrm{ox}}\right)$, deoxygenated $\left(\mathrm{Hb}_{\mathrm{deox}}\right)$, oxygen saturation, and total hemoglobin. In this study, the $\mathrm{Hb}_{\mathrm{ox}}$ was chosen as the target of interest because it expresses the result of oxygen consumption and perfusion. The optodes were placed on the skin overlying the medial head of the GAS muscle and the proximal part of the anterior tibial (TA) muscle using the patients most symptomatic and most ischemic leg. The placement of the optode on the medial head of the GAS muscle was chosen to increase comparability with earlier studies. ${ }^{8}$ The proximal part of the TA muscle was chosen because this area of the muscle has the largest cross-sectional area and contains less connective tissue and tendons. In patients without PAD, the right leg was used. Both optodes were placed on a circumferential line around the calf $10 \mathrm{~cm}$ below the tuberosity of the tibial bone. The NIRS optodes were held in place by elastic bandage.

Treadmill exercise protocol: The patient was placed on a treadmill with the NIRS optodes placed as described above.
The walking speed was set at $2.4 \mathrm{~km} / \mathrm{h}$ with an inclination of $12 \%$ after an initial resting period of 3 minutes as previously done. $^{3,8}$ The patients continued to walk until the calf pain made them discontinue or the tests were terminated after 5 minutes (distance $200 \mathrm{~m}$ ). Measurements continued afterwards for a period of 6 to 10 minutes until the NIRS parameters had recovered.

The experimental setup is shown in - Fig. 1 and an example of both tests is shown in -Figs. 2 and $\mathbf{3}$.

\section{Calculations and Statistics}

All fittings and graphs were made using GraphPad Prism 5 (San Diego, CA).

The area under the $\mathrm{Hb}_{\mathrm{ox}}$ curve was used to compare the oxygen decrease during exercise. The initial slope of the recovery curve was determined using a linear regression model to describe the initial velocity of the recovery of $\mathrm{Hb}_{\mathrm{ox}}$, like previously done by Kemp et al. ${ }^{10}$ The kinetics of the NIRS- $\mathrm{Hb}_{\mathrm{ox}}$ recovery curve has a fairly constant initial velocity until reaching a plateau. In some cases, an exponential fit was more suitable but unfortunately this is not always the case. Some patients have different recovery curve shapes according to the degree of disease, ${ }^{8}$ which makes a linear model more suitable. The intercept with the $x$-axis of the fitted line is the assumed recovery time. Reliability for the recovery periods in both exercise tests was obtained by comparing the slopes of the recovery linear fit.

The force measurements obtained during pedal exercise were expressed as impulse $(\mathrm{N} \times \mathrm{s})$, which is a surrogate for workload in isometric exercise. The impulse was calculated using the voltage output from the strain gauge calibrated with a $10 \mathrm{~kg}$ weight multiplied with the exercise duration in seconds and the acceleration of gravity (mean $\mathrm{kg}$ in the exercise period $\times 60$ seconds $\times 9.807 \mathrm{~m} / \mathrm{s}^{2}=$ impulse $\mathrm{N} \cdot \mathrm{s}$. 


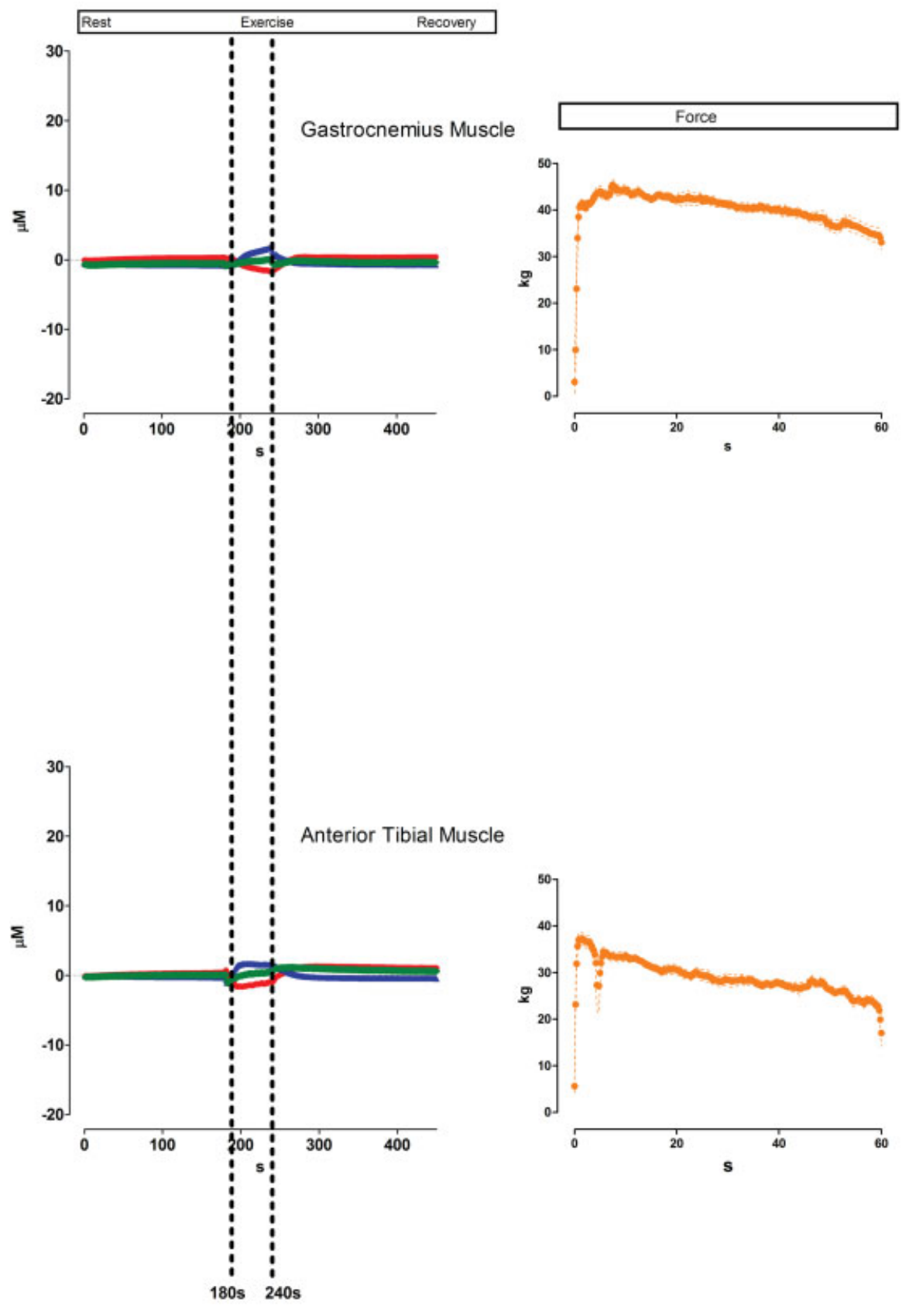

\section{- $\mathrm{Hb}$ ox $\quad \mathrm{Hb}$ deox $\quad \mathrm{Hb}$ total $\quad$ Force $\mathrm{kg}$}

Fig. 2 Control experiment with the pedal test using one of the authors (B.L.P.) as a subject (each curve consists of six replicates).

One way, ANOVA and ICC were used to describe reproducibility. ${ }^{10,14}$ The ICC is a way to incorporate both within and between people variability. Statistical significance was defined as a $p$-value $<0.05$. All statistics were done using SPSS 20.0 (IBM, Chicago, IL).

\section{Results}

In all patients and controls, a decrease in $\mathrm{Hb}_{\mathrm{ox}}$ was observed during exercise in both muscles. An increase in $\mathrm{Hb}_{\mathrm{deox}}$ during exercise was seen for all patients in the pedal test. In the treadmill protocol, the same response was true for patients with PAD, but in the experiments on the controls, including one of the authors (BLP), it either decreased in the GAS muscle or stabilized on a low level in the TA muscle (see - Figs. 2, 3, and $\mathbf{4}$ for experiment examples).

The ICC of the GA muscle was between 0.92-0.95 (footpedal) and $0.70-0.98$ (treadmill) and the anterior tibial muscle was between 0.87-0.96 (foot-pedal) and 0.67-0.79 (treadmill) (-Table 2). Reliability using ICC values and other 

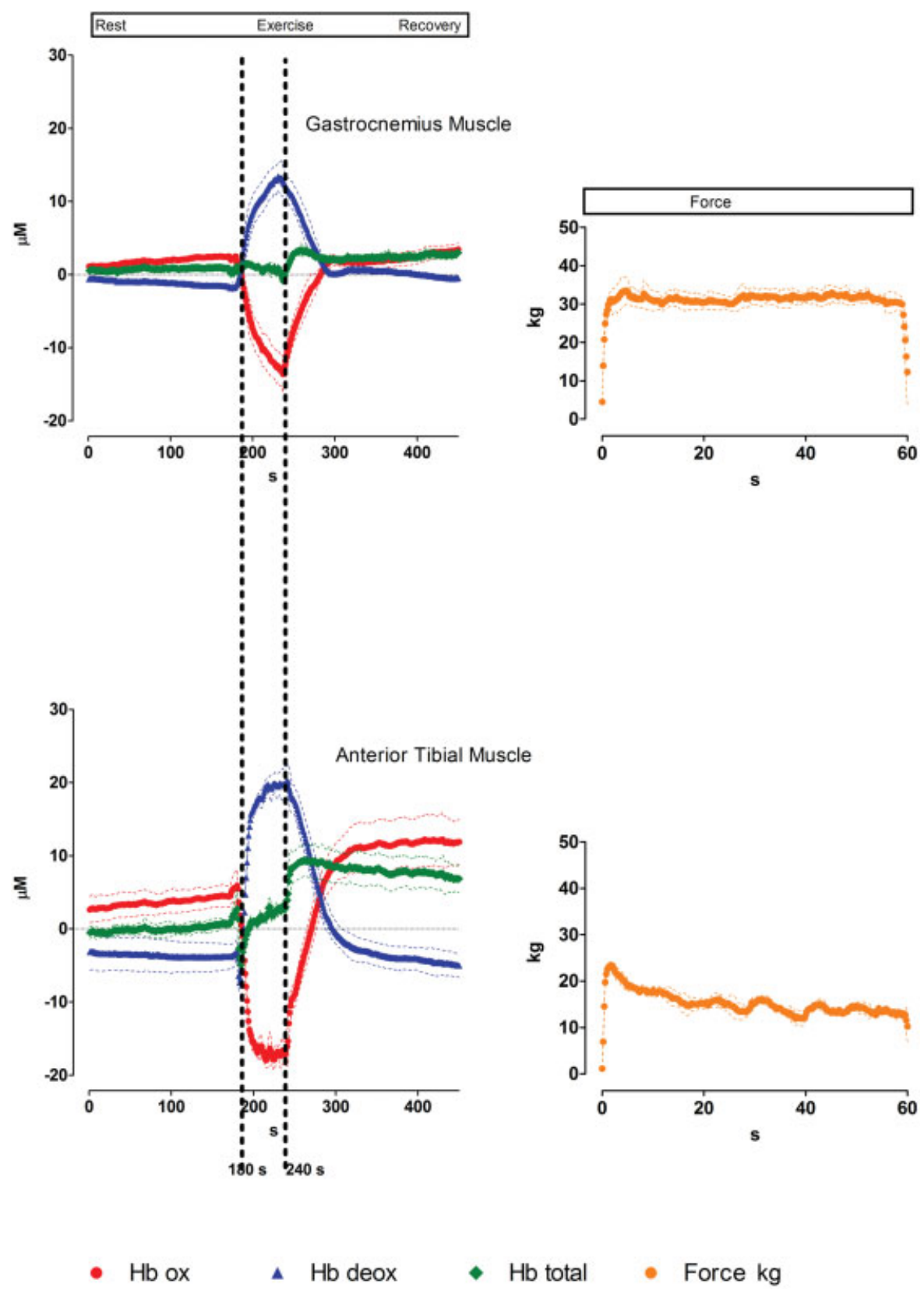

Fig. 3 Example of experiment with the pedal test using a patient with peripheral arterial disease (each curve consists of three replicates).

reliability values, for example, Cronbach $\alpha$ value, is generally considered excellent $>0.80$ and fair $>0.60$. This places the values obtained in this study in the high end of the scale for both tests. ${ }^{3,8}$

Generally, reproducibility was lower in the treadmill test compared with the pedal test in both the exercise and recovery situations, both due to wide $95 \%$ confidence intervals of the ICC values in the treadmill test no significant ( $p>0.05$ ) difference could be established. The reliability of the walking time was high but is lower in reality because the treadmill protocol was terminated at $200 \mathrm{~m}$. The reliability of muscle force measured as impulse was high in the pedal test. No difference in the reproducibility was found between the GAS and the TA muscles for the pedal test except for a slightly lower ICC for the TA muscle in the force measurements, although not significant. In the treadmill test, a lower ICC was seen in the exercise period for the TA muscle but was opposite in the recovery period. The ICC reliability results are summarized in -Table 2 and the $\mathrm{Hb}_{\mathrm{ox}}$ data and calculated kinetics are shown in - Table 3.

\section{Discussion}

The findings of this study show superior reproducibility of the isometric NIRS pedal test compared with the NIRS treadmill tests (ICC GAS muscle 0.92-0.95 [foot-pedal] and 0.70-0.98 [treadmill], TA muscle $0.87-0.96$ [foot-pedal] and 0.67-0.79 [treadmill]), even though no significant difference is detected 


\section{Control}

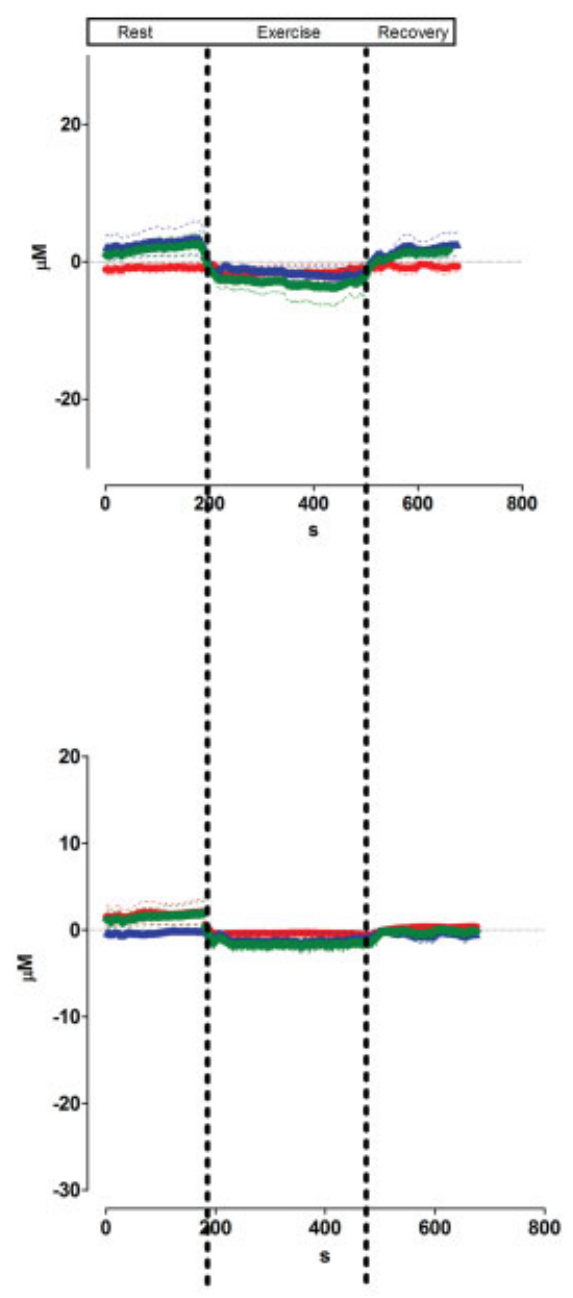

- Hb ox
PAD

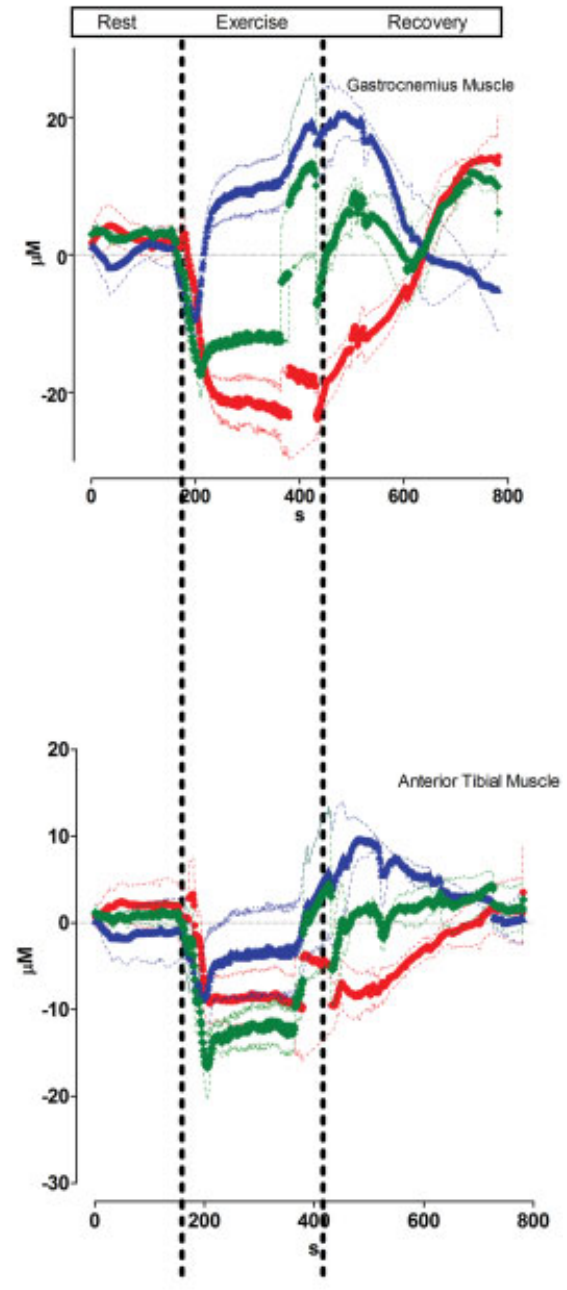

- $\mathrm{Hb}$ total

Fig. 4 Near infrared spectroscopy data from the treadmill test using one of the authors (control) $(n=6)$ and a patient with peripheral arterial disease $(n=3)$.

because of the large confidence intervals of the treadmill test. But this fact in itself indicates a wider range of measures in the treadmill test. This is likely to be a reflection of factors described in the next section.

\section{Area under the Curve}

The difference in ICC values between the pedal test and the treadmill test in this parameter is seen in the TA muscle where the difference is approximately $15 \%$ opposed to the GAS muscle where it was only $8 \%$ (-Table 2 ). This difference could be explained by a smaller decrease in $\mathrm{Hb}_{\mathrm{ox}}$ in the $\mathrm{TA}$ muscle during walking compared with the isometric exercise which could compromise reproducibility.

The use of area under the decreasing $\mathrm{Hb}_{\mathrm{ox}}$ NIRS curve (AUC) in the exercise period was considered as a fair method to describe reproducibility. Another method using the initial velocity of the oxygen decrease during exercise could have been used. But AUC was chosen because it omits the exercise duration where the initial velocity does not.

\section{The Near Infrared Spectroscopy Recovery Curve}

Generally, the NIRS recovery kinetics reflects the quality of the revascularization after exercise.

The difference in ICC values between the two tests was still highest in the pedal test for both muscles, but during recovery the highest difference was seen in the GAS muscles (15\%) and the lowest in the TA muscles (6\%), which is the opposite response compared with the AUC during exercise. The explanation for this might be the same as for the AUC curve, but it is not clear.

The interpretation of recovery curve in the treadmill test in patients without PAD is somewhat different compared to 
Table 2 Intraclass correlation constant values of reproducibility (95\% confidence intervals; $N=11$ )

\begin{tabular}{|c|c|c|c|c|}
\hline & \multicolumn{2}{|c|}{ Pedal instrument } & \multicolumn{2}{|l|}{ Treadmill } \\
\hline & Gastrocnemius & Anterior tibial & Gastrocnemius & Anterior tibial \\
\hline Exercise (AUC) & $\begin{array}{l}0.936 \\
0.828-0.981\end{array}$ & $\begin{array}{l}0.927 \\
0.804-0.979\end{array}$ & $\begin{array}{l}0.852 \\
0.602-0.956\end{array}$ & $\begin{array}{l}0.672 \\
0.113-0.903\end{array}$ \\
\hline Initial recovery velocity & $\begin{array}{l}0.953 \\
0.873-0.986\end{array}$ & $\begin{array}{l}0.956 \\
0.880-0.987\end{array}$ & $\begin{array}{l}0.701 \\
0.192-0.912\end{array}$ & $\begin{array}{l}0.792 \\
0.440-0.939 .\end{array}$ \\
\hline Walking time & & & \multicolumn{2}{|l|}{$\begin{array}{l}0.983 \\
0.954-0.995\end{array}$} \\
\hline Muscle force (impulse) & $\begin{array}{l}0.920 \\
0.785-0.976\end{array}$ & $\begin{array}{l}0.866 \\
0.639-0.961\end{array}$ & & \\
\hline
\end{tabular}

Abbreviation: AUC, area under curve.

patients with PAD, because some of these patients have already recovered their $\mathrm{Hb}_{\mathrm{ox}}$ within the exercise period. This is explained by the compensatory blood flow response to exercise. ${ }^{15}$ In patients with PAD, the blood flow response is impaired due to the stiffness of the vascular bed which makes the vessels less compliant and, consequently, the normal blood flow response to exercise is insufficient. ${ }^{16}$ In patients with PAD, the recovery curve never recovers within the exercise period on the treadmill. If a patient with PAD has a more horizontal slope of the recovery curve, it means that they will have a longer recovery time and hereby be more severely affected by their disease. ${ }^{8}$ If a person without PAD has a horizontal slope of their recovery curve it will have the opposite meaning. However, this phenomenon is not seen when using the pedal test. The pedal test is, therefore, properly more feasible to differentiate between mild and no claudication. The reproducibility of the NIRS treadmill test was lower in this study compared with results achieved by, for example, Komiyama who reported ICC values of 0.92 in 21 legs in 11 patients. Komiyama et al had a narrower range in intervals between measurements which was 6 days (mean, range 3-9 days). Interval between measurements was 22.6 days (mean, range 2-130 days) in this study and could explain the lower reproducibility; however, if this was the case one would have expected the same low reproducibility for the pedal test, which clearly was not the case. Komiyama ${ }^{8}$ used recovery time and not initial recovery velocity in this study which could again explain the difference.

\section{Muscle Force and Walking Time}

These two parameters are an expression of the amount of muscle work done in the two tests. Both show high reproducibility values. The highest is seen in the walking time but these values may be falsely elevated because of the termination of the treadmill test, which is set at $200 \mathrm{~m}$.

Equal ICC values are seen between the two muscle types in both tests.

\section{Body Mass Index and Physical Fitness}

Body mass index (BMI) and physical fitness contribute to the intersubject variability of whole body exercise more than a single-joint exercise. BMI varies among patients involved in this study with a SD of $\pm 18 \%(25 \pm 4.6)$. Variability of the BMI is not reported in the cited articles involving treadmill exercise. Therefore, the impact of BMI on their results cannot be studied. Whole body physical fitness in this study was not measured and walking time/distance cannot be used as a true reflection of variability because the treadmill test was terminated at $200 \mathrm{~m}$. In general among PAD patients, a coefficient of variability (CV) of $43 \%$ was seen in walking distance in a recent study with GPS-monitored walking distance. ${ }^{17}$

The data obtained with one of the authors (B.L.P.) as a subject were included to illustrate the data with a younger, fit person well acquainted with the instrument and the procedure. These control experiments in fact scored the highest reproducibility (see - Figs. 2 and $\mathbf{4}$ ).

Table 3 Calculated NIRS-kinetics from $\mathrm{Hb}_{\mathrm{ox}}$ of all patients $(N=11 ; \pm \mathrm{SD})$

\begin{tabular}{|l|l|l|l|l|}
\hline \multicolumn{2}{|l|}{} & \multicolumn{2}{l|}{ Pedal instrument } & \multicolumn{2}{l|}{ Treadmill } \\
\cline { 2 - 5 } & Gastrocnemius & Anterior tibial & Gastrocnemius & Anterior tibial \\
\hline \multicolumn{2}{|l|}{ Oxy hemoglobin data for all subjects $(\boldsymbol{n}=11)$} & $153.0 \pm 129.2$ & $302.9 \pm 261.8$ & $302.9 \pm 261.8$ \\
\hline AUC exercise & $302.9 \pm 261.8$ & $0.29 \pm 0.24$ & $0.13 \pm 0.09$ & $0.05 \pm 0.05$ \\
\hline Initial recovery velocity & $0.25 \pm 0.21$ & $6,970.1 \pm 2,821.1$ & & $233.3 \pm 80.7$ \\
\hline $\begin{array}{l}\text { Force (impulse) } \\
(\mathrm{N} \times \mathrm{s})\end{array}$ & $13,222.8 \pm 6,171.0$ & & & \\
\hline Walking time $(\mathrm{s})$ & & & \\
\hline
\end{tabular}

Abbreviations: AUC, area under curve; $\mathrm{Hb}_{\mathrm{ox}}$, oxygenated hemoglobin; NIRS, near infrared spectroscopy; SD, standard deviation. 
The observed curve patterns between the different parameters of hemoglobin are in concordance with the earlier findings of NIRS experiments of patients with PAD

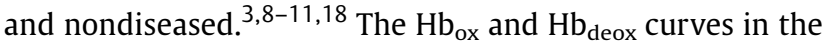
pedal test were a mirror reflection of each other in both the patients with and without PAD, but the span of the patients with PAD was 10 to 15 times higher (-Figs. 2 and 3). The $\mathrm{Hb}_{\text {total }}$ increased in the controls during exercise but kept constant in the patients with PAD. This could be explained by the stiffness of the large arteries in the leg in the patients with PAD impairing the normal increase in perfusion by arterial dilatation during exercise. ${ }^{15}$ During recovery, an elevated $\mathrm{Hb}_{\text {total }}$ is seen in all patients and is the result of the postischemic hyperaemia, ${ }^{15}$ which also shifts hemoglobin balance towards a greater amount of $\mathrm{Hb}_{\mathrm{ox}}$ compared with $\mathrm{Hb}_{\text {deox}}$.

During the treadmill exercise, the response was different. In the experiments using one of the authors, an initial drop in both $\mathrm{Hb}_{\mathrm{ox}}$ and $\mathrm{Hb}_{\mathrm{deox}}$ was seen in both muscles. The starting level of $\mathrm{Hb}_{\mathrm{deox}}$ and $\mathrm{Hb}_{\text {total }}$ was high, properly reflecting the pooling of blood in the legs in the standing position. ${ }^{19}$ In the GAS muscle, a slight increase of $\mathrm{Hb}_{\mathrm{ox}}$ and a slight decrease of $\mathrm{Hb}_{\text {deox }}$ and $\mathrm{Hb}_{\text {total }}$ were seen during exercise. In the $\mathrm{TA}$ muscle, all values stabilized on a constant level. During recovery, the same response as for the pedal protocol was seen.

The explanation for the observed difference in $\mathrm{Hb}_{\mathrm{ox}}$, $\mathrm{Hb}_{\mathrm{deox}}$, and $\mathrm{Hb}_{\text {total }}$ between the pedal and treadmill test during exercise should be found in the difference of isometric and dynamic exercise types. In the isometric exercise, the perfusion is blocked during exercise because of the constant contraction in the whole exercise period. In the treadmill test, perfusion is not blocked in the same way because of the intermittent contraction during walking, allowing perfusion during muscle relaxation.

Statistical Evaluation of Reproducibility of Measurements The use of ICC in this study was chosen as a key parameter because of its common use in previous studies that make the ICC more comparable even though ICC values from different studies are not really comparable unless a similar study design is applied. ${ }^{20,21}$

The excellent ICC of the pedal test is high compared with the dynamic foot pedal test presented by Yamamoto et al and Manning et al., ${ }^{3,12}$ No real difference in reproducibility was found between the GAS and TA muscles except for a lower reproducibility for the TA muscle during exercise in the treadmill protocol ( $\mathbf{- T a b l e ~} \mathbf{2}$ ).

The simplicity and usefulness of the pedal test renders it as a candidate of standard test in the PAD clinic as demonstrated in this study demonstrating high reproducibility of PAD evaluation. Thus, it would seem worthwhile investigating the method further on a larger number of PAD patients focusing also on a model for disease sensitivity and specificity. This could be achieved by setting up a randomized study where patients are randomized to a treadmill test or to the isometric pedal test, where the treadmill test is considered the golden standard. Resting ABI could also be chosen as the golden standard. Interobserver agreement should also be considered because all tests were done by the same person in this study. The result of such a study will properly show lower reproducibility like in other clinical tests where several persons perform the same test. ${ }^{22}$

Calf pain in PAD patients is predominately located in the GAS muscles and almost never in the TA muscles, which also is used during walking and represents the agonistic muscle force to the GAS muscle. Furthermore, the TA muscle as opposed to the GAS muscle is mainly an oxidative muscle in humans containing 69\% type I and $27 \%$ type Ila fibers. ${ }^{23,24}$ To elaborate whether the measurement in the TA muscle has equal reproducibility, we tested this muscle.

In summary, the new pedal test shows high reproducibility, are easier to complete for both investigator and patient compared with older ABI-based protocols. The contribution of the BMI on the in between subject variability are reduced. Muscle oxygen consumption is omitted in the determination of the disease stage, which is left out using ankle pressures. Using NIRS, continuous measurements are provided; this is impossible using $\mathrm{ABI}$ measurements. Compared with the NIRS treadmill test, the reliability is slightly higher, the pedal test can be used on patients suffering from critical ischemia which the NIRS treadmill test. The pedal test are most likely better at separating muscle ischemia due to PAD from other comorbidities influencing walking distance when these diseases equally effect walking.

\section{Conclusion}

This study describes a new, simple pedal apparatus and test designed to evaluate the level of the PAD disease as well as the effects of various treatment procedures applied to these patients. The new pedal test was compared with an existing treadmill test. The pedal test proved easy to carry out and was well tolerated by the patients. Also, the pedal test showed higher reproducibility of test results on the same patient than the treadmill test.

The test procedure developed remains, however, to be applied in a larger group of patients.

\section{Acknowledgments}

This project is part of a Ph.D. thesis authored by B.L.P. and it was supported by Aase and Ejnar Danielsens Foundation, Snedkermester Sophus Jacobsen, and hustru Astrid Jacobsens Foundation, The Hartmann Foundation and the Danish capital regional administration. Thanks should be given to Mr. Palle Kock and Mr. Ib Terkelsen for technical help with equipment design and construction.

\section{References}

1 Norgren L, Hiatt WR, Dormandy JA, et al; TASC II Working Group. Inter-Society Consensus for the Management of Peripheral Arterial Disease (TASC II). Eur J Vasc Endovasc Surg 2007;33(Suppl 1):S1-S75

2 Gardner AW, Afaq A. Management of lower extremity peripheral arterial disease. J Cardiopulm Rehabil Prev 2008;28(6):349-357 
3 Yamamoto K, Miyata T, Onozuka A, Koyama H, Ohtsu H, Nagawa H. Plantar flexion as an alternative to treadmill exercise for evaluating patients with intermittent claudication. Eur J Vasc Endovasc Surg 2007;33(3):325-329

4 Cameron AE, Porter A, Rosser S, Da Silva AE, De Cossart LM. The Stresst'er ergometer as an alternative to treadmill testing in patients with claudication. Eur J Vasc Endovasc Surg 1997;14(6):433-438

5 Chance B, Dait MT, Zhang C, Hamaoka T, Hagerman F. Recovery from exercise-induced desaturation in the quadriceps muscles of elite competitive rowers. Am J Physiol 1992;262(3 Pt 1):C766-C775

6 Rivera-Brown AM, Frontera WR. Principles of exercise physiology: responses to acute exercise and long-term adaptations to training. PM R 2012;4(11):797-804

7 Pipinos II, Judge AR, Zhu Z, et al. Mitochondrial defects and oxidative damage in patients with peripheral arterial disease. Free Radic Biol Med 2006;41(2):262-269

8 Komiyama T, Shigematsu H, Yasuhara H, Muto T. Near-infrared spectroscopy grades the severity of intermittent claudication in diabetics more accurately than ankle pressure measurement. $\mathrm{Br}$ J Surg 2000;87(4):459-466

9 Watanabe T, Matsushita M, Nishikimi N, Sakurai T, Komori K, Nimura Y. Near-infrared spectroscopy with treadmill exercise to assess lower limb ischemia in patients with atherosclerotic occlusive disease. Surg Today 2004;34(10):849-854

10 Kemp GJ, Roberts N, Bimson WE, et al. Mitochondrial function and oxygen supply in normal and in chronically ischemic muscle: a combined 31P magnetic resonance spectroscopy and near infrared spectroscopy study in vivo. J Vasc Surg 2001;34(6):1103-1110

11 McCully KK, Halber C, Posner JD. Exercise-induced changes in oxygen saturation in the calf muscles of elderly subjects with peripheral vascular disease. J Gerontol 1994;49(3):B128-B134

12 Manning BJ, McGreal G, Crowley H, Redmond HP, O’Donnell JA. A prospective comparison of pedal ergometry with conventional treadmill testing in the investigation of lower extremity pain. Ir J Med Sci 2001;170(3):169-171
13 Quistorff B, Nielsen S, Thomsen C, Jensen KE, Henriksen O. A simple calf muscle ergometer for use in a standard whole-body MR scanner. Magn Reson Med 1990;13(3):444-449

14 Petrie A, Sabin C. Medical Statistics at a Glance. 3rd ed. Oxford England: Wiley-Blackwell; 2009

15 Casey DP, Joyner MJ. Local control of skeletal muscle blood flow during exercise: influence of available oxygen. J Appl Physiol (1985) 2011;111(6):1527-1538

16 Taniwaki H, Shoji T, Emoto M, et al. Femoral artery wall thickness and stiffness in evaluation of peripheral vascular disease in type 2 diabetes mellitus. Atherosclerosis 2001;158(1):207-214

17 Le Faucheur A, Noury-Desvaux B, Mahé G, et al. Variability and short-term determinants of walking capacity in patients with intermittent claudication. J Vasc Surg 2010;51(4):886-892

18 Bauer TA, Brass EP, Barstow TJ, Hiatt WR. Skeletal muscle StO2 kinetics are slowed during low work rate calf exercise in peripheral arterial disease. Eur J Appl Physiol 2007;100(2):143-151

19 Truijen J, Kim YS, Krediet CT, et al. Orthostatic leg blood volume changes assessed by near-infrared spectroscopy. Exp Physiol 2012; 97(3):353-361

20 Rankin G, Stokes M. Reliability of assessment tools in rehabilitation: an illustration of appropriate statistical analyses. Clin Rehabil 1998;12(3):187-199

21 Bland JM, Altman DG. Agreement between methods of measurement with multiple observations per individual. J Biopharm Stat 2007;17(4):571-582

22 Sierra F, Cárdenas A. Evidence-based medicine (EBM) in practice: agreement between observers rating esophageal varices: how to cope with chance? Am J Gastroenterol 2007;102(11):2363-2366

23 Nakagawa Y, Ratkevicius A, Mizuno M, Quistorff B. ATP economy of force maintenance in human tibialis anterior muscle. Med Sci Sports Exerc 2005;37(6):937-943

24 Askew CD, Green S, Walker PJ, et al. Skeletal muscle phenotype is associated with exercise tolerance in patients with peripheral arterial disease. J Vasc Surg 2005;41(5):802-807 